\title{
INVESTIGACIÓN
}

Recibido: 07/06/2019 --- Aceptado: 17/12/2019 --- Publicado: 15/06/2020

\section{ENTORNO VIRTUAL E-EVALUACIONES COMO HERRAMIENTA DE GESTIÓN EN GRUPOS NUMEROSOS}

\section{Virtual environment e-evaluation as a management tool in numerous groups}

(iD) $R^{G}$ Piero Espino Román1. Universidad Politécnica de Sinaloa. México. pespino@upsin.edu.mx

Eugenia Olaguez Torres. Universidad Politécnica de Sinaloa. México. jolaguez@upsin.edu.mx

Ricardo Alonso López Hernández. Universidad de Politécnica de Sinaloa. México.

$\underline{2016030975 @ u p s i n . e d u . m x}$

Claudio Rafael Vásquez Martínez. Universidad de Guadalajara. México. crvasquezm@gmail.com

\section{RESUMEN}

En este artículo se presenta el desarrollo de un entorno virtual de e-evaluaciones para la administración de evaluaciones y seguimiento del rendimiento académico de los estudiantes en grupos numerosos, aplicado en una prueba piloto en la unidad académica de ingeniería mecatrónica de la Universidad Politécnica de Sinaloa, México. El desarrollo del entorno virtual de e-evaluaciones consiste en el uso del framework de modelo-vista-controlador, CodeIgniter desarrollado por EllisLab, al mismo tiempo se utilizó Bootstrap un framework desarrollado dentro de la compañía Twitter con el objetivo de estandarizar las herramientas que se utilizan en el desarrollo del front-end. Los resultados obtenidos permiten observar un incremento en la interacción sincrónica y asincrónica entre docente y estudiante en un entorno virtual e-evaluaciones; por su parte de los docentes se midió y se observó un ahorro en el tiempo destinado a realizar la evaluación en grupos numerosos, se realizó la evaluación a través de una administración personalizada de cada uno de los estudiantes, por otro lado el entorno virtual hizo partícipe al estudiante de su propia evaluación y retroalimentación, la experiencia en el uso del entorno virtual dicha por los alumnos, fue positiva dado que les permitió desarrollar la evaluación de una manera interactiva en un menor tiempo. Como conclusión se obtuvo que el entorno

\footnotetext{
1 Piero Espino Román: Unidad académica de ingeniería mecatrónica e investigación y posgrado, Universidad Politécnica de Sinaloa, Mazatlán, Sinaloa, México.
} 
Espino Román, P., Olaguez Torres, E., López Hernández, R. A. y Vásquez Martínez C. R. Entorno virtual e-evaluaciones como herramienta de gestión en grupos numerosos

virtual e-evaluaciones aporta elementos que promueve el seguimiento y monitoreo de los resultados de la evaluación.

PALABRAS CLAVE: e-evaluación - entorno virtual - grupos numerosos herramientas web - educación a distancia - evaluación - educación superior aprendizaje.

\section{ABSTRACT}

This article presents the development of a virtual environment of e-evaluations for the management of evaluations and monitoring of the academic performance of students in large groups, applied in a pilot test in the academic unit of mechatronic engineering of Sinaloa Polytechnic University, Mexico. The development of the virtual environment of e-evaluations consists in the use of the model-view-controller framework, CodeIgniter developed by EllisLab, at the same time Bootstrap, a framework developed within the Twitter company, was used with the aim of standardizing the tools that are used in the development of the front-end. The results obtained allow us to observe an increase in synchronous and asynchronous interaction between professor and student in a virtual environment of e-evaluations; for their part, the professors measured and observed a saving in the time allocated to carry out the evaluation in large groups. The evaluation was carried out through customized management of each of the students. On the other hand, the virtual environment involved the student in his own evaluation and feedback, the experience in the use of the virtual environment said by the students was positive since it allowed them to develop the evaluation in an interactive way in a shorter time. In conclusion, it was obtained that the virtual e-evaluations environment provides elements that promote the follow-up and monitoring of the evaluation results.

KEYWORDS: e-evaluation - virtual environment - numerous groups - wed tools distance education - evaluation - higher education - learning.

\section{ENTORNO VIRTUAL, AVALIAÇÕES ONLINE COMO FERRAMENTA DE GESTÃO EM GRUPOS NUMEROSOS}

\section{RESUMO}

Neste artigo se apresenta o desenvolvimento de um entorno virtual de avaliações online para a administração de avaliações e seguimento do rendimento acadêmico dos alunos em grupos numerosos, aplicado numa prova piloto na unidade acadêmica de engenharia mecatrônica da Universidade Politécnica de Sinaloa, México. O desenvolvimento do entorno virtual de avaliações online consiste no uso do framework do modelo-vista-controlador, CodeIgniter desenvolvido pelo EllisLab, ao mesmo tempo se utilizou Bootstrap um framework desenvolvido dentro da empresa Twitter com o objetivo de padronizar as ferramentas que se usam no 
Espino Román, P., Olaguez Torres, E., López Hernández, R. A. y Vásquez Martínez C. R. Entorno virtual e-evaluaciones como herramienta de gestión en grupos numerosos

desenvolvimento do front-end. Os resultados obtidos permitem observar um incremento na interação síncrona e assíncrona entre professor e aluno em um ambiente virtual de avaliação online; por parte dos professores se mensurou e se observou uma economia no tempo destinado a fazer a avaliação em grupos numerosos, se executou a avaliação através de uma administração personalizada de cada um dos alunos, por outro lado o entorno virtual fez participar ao aluno da sua própria avaliação e retroalimentação, a experiência no uso do entorno virtual de acordo com os alunos, foi positiva dado que permitiu-lhes desenvolver a avaliação de forma interativa em um tempo mais curto. Como conclusão se obteve que o entorno virtual e as avaliações online aportam elementos que promovem $\mathrm{o}$ seguimento e monitoramento dos resultados da avaliação.

PALAVRAS CHAVE: avaliações online - entorno virtual - grupos numerosos ferramentas web - educação a distância - avaliação - educação superior aprendizado.

\section{Cómo citar el artículo:}

Espino Román, P., Olaguez Torres, E., López Hernández, R. A. y Vásquez Martínez C. R. (2020). Entorno virtual e-evaluaciones como herramienta de gestión en grupos numerosos. [Virtual environment e-evaluation as a management tool in numerous groups]. Vivat Academia. Revista de Comunicación, (151), 107-125.

doi: http:// doi.org/10.15178/va.2020.151.107-125 Recuperado de

http://www.vivatacademia.net/index.php/vivat/article/view/1201

\section{INTRODUCCIÓN}

Tanto en México como a nivel global, las agendas políticas presentan una mayor participación en temas relacionados con educación, en donde se refleja que impera mayormente la cobertura en educación. En el Plan Nacional de Desarrollo (20132018) del gobierno de la república en México, estableció en una de sus líneas de acción, alcanzar la cobertura de la educación superior en al menos un $40 \%$ para el año 2018 (González, 2015). Respecto a la cobertura de la educación superior la Asociación Nacional de Universidades e Instituciones de Educación Superior (ANUIES) ha propuesto la meta de aumentar la cobertura de 60 por ciento en el ciclo 2021-2022, es decir, una matrícula de 5 millones 840 mil estudiantes, por tanto, la matrícula debería aumentar en 257 mil cada año. En total, la matrícula se incrementa más de 2 millones 570 mil adicionales a los registrados en el ciclo 2011-2012 (Arias et al., 2013). En el ciclo escolar 2016-2017, en educación superior, de acuerdo con la Secretaría de Educación Pública (2017) y con base al informe que presentó la Organización para la Cooperación y el Desarrollo Económico (OCDE) indica que en el ciclo escolar 2016-2017, incrementó con una cobertura total de 5.2 puntos porcentuales, de $32.1 \%$ a $37.3 \%$.

En el caso particular del Estado de Sinaloa, México, la cobertura de educación superior se encuentra situado en el primer lugar a nivel nacional con un $43 \%$ esto en 
Espino Román, P., Olaguez Torres, E., López Hernández, R. A. y Vásquez Martínez C. R. Entorno virtual e-evaluaciones como herramienta de gestión en grupos numerosos

el ciclo escolar 2015-2016 de acuerdo con estadísticas de la Secretaría de Educación Pública (2016). La Universidad Politécnica de Sinaloa, México, en los últimos años ha tenido un aumento considerable en la matrícula de acuerdo con la Agenda Estadística Upsin (2017). En relación con lo mencionado, la Universidad Politécnica de Sinaloa en el ciclo escolar 2017-2018, siendo el programa educativo de Ingeniería Mecatrónica el que registra mayor matrícula. Desde el inicio de la Institución Educativa en el ciclo escolar 2005-2006, Ingeniería Mecatrónica registró una matrícula total de 70 estudiantes en comparación al ciclo escolar 2017-2018 que registra un total de 654 estudiantes que se encuentran matriculados, lo que representa un incremento porcentual de $934 \%$ a lo largo de 12 años.

De tal manera que teniendo la determinación de cumplir con la meta planteada en la cobertura de educación superior en las Instituciones de Educación Superior (IES) ha provocado notablemente que se genere el efecto de sobrepoblación en grupos, generando que cada vez los grupos sean más numerosos. Aguerrondo (2008) señala que la gran cantidad de estudiantes en las aulas se genera por el deseo de tener una alta cobertura en la educación y esto produce el tener que realizar un cambio significativo en las propuestas de enseñanza-aprendizaje y produce un deterioro en la calidad educativa. Diversos estudios han detectado que un factor que definitivamente incide en el rendimiento académico de los estudiantes son las aulas numerosas. Gutiérrez (2000) muestran en su estudio que debido al gran número de estudiantes por grupo, hay poco o nulo intercambio de experiencias didácticas entre docentes y estudiantes.

Por otro lado, Mendoza (2013) menciona que un factor que juega un papel importante en las aulas numerosas es la infraestructura ya que, si no se cuenta con un espacio suficiente, es difícil impartir clases y desarrollar capacidades y habilidades en los estudiantes. Ocasionando que los docentes tienden a centrarse más en el control de la disciplina y la interacción del estudiante y docente se ve limitada a una falta de comunicación y escaso tiempo para realizar evaluaciones de forma individual por parte del docente (Rocha, 2017; Fondón et al., 2010). Además como lo señala Rodríguez y Díaz (2015) y Sobrados (2016), para el docente de tener grupos grandes en el aula, reside en la carga excesiva de trabajo que se concreta en: distribuir y recoger tareas, pruebas, realizar seguimiento y tutorías, corregir, calificar y suscitar retroalimentación en los trabajos de los estudiantes. Como se ha señalado el incremento de grupos numerosos conlleva un gran desafío para el docente al multiplicarse su carga de trabajo por lo que se deben de establecer estrategias que faciliten esta labor sobre todo el introducir procedimientos de evaluación y retroalimentación en masa. Factor que constituye el problema de esta investigación. Que mereció un ejercicio académico de búsqueda de estrategia didáctica en la optimización de la gestión del proceso de evaluación en grupos con una permanente sobrepoblación de estudiantes.

Existen diversas estrategias que se apoyan el uso de entornos virtuales de aprendizaje como una opción para atender el mayor número de estudiante, los entornos virtuales consisten en un espacio educativo alojado en la web y conformado 
Espino Román, P., Olaguez Torres, E., López Hernández, R. A. y Vásquez Martínez C. R. Entorno virtual e-evaluaciones como herramienta de gestión en grupos numerosos

por un conjunto de herramientas informáticas que posibilitan la interacción didáctica (Salinas, 2011). Cuesta y Alegre (2011), señalan que los entornos virtuales de aprendizaje se presentan como una herramienta realmente útil a la hora de abordar el reto de la evaluación continuada en grupos numerosos. Lara (2001) indica que es un entorno de enseñanza-aprendizaje basado en aplicaciones telemáticas, en el cual interactúa la informática y los sistemas de comunicación. Los entornos virtuales ofrecen una alternativa viable para evaluar el desempeño académico, tanto individual como grupal, al facilitar el registro y tratamiento de datos, para ser utilizados como evidencias confiables en el proceso de evaluación (Cano y Hernández, 2009). Un entorno virtual tiene la ventaja que se convierte en un espacio para la transmisión de información, ya que los profesores proporcionan a los estudiantes documentos, lecturas y enlaces (Santibáñez, 2010). Los entornos virtuales de aprendizaje contribuyen una forma totalmente nueva de tecnología educativa ofreciendo la disponibilidad de varias estrategias para fomentar el aprendizaje complejo a partir de la utilización y vinculación de las tecnologías de la información y comunicación (TIC) como los blogs, foros de discusión, las redes sociales, los chats y los entornos virtuales de aprendizaje (Martínez et al., 2013 y Murrieta, 2016).

Una alternativa de entornos virtuales son el uso de plataforma e-evaluaciones permiten la interacción docente y estudiante de manera online. La e-evaluación se entiende como cualquier proceso electrónico de evaluación en el que son utilizadas las TIC para la presentación de las actividades, las tareas de evaluación y el registro de las respuestas (Chiva et al., 2013; Rodríguez et al., 2011). Bretones (2008) las describe como una evaluación continua donde participa el estudiante en su propia evaluación. La e-evaluación se ha convertido en un aspecto nuclear para las universidades que desarrollan sus programas educativos bajo entornos en línea (Hernández et al. 2018). Las e-evaluaciones es un componente cada vez más importante en los cursos en línea (Wambui et al., 2014; Espino et al., 2019). Ofreciendo diversos beneficios tanto como para el estudiante y docente. Entre los que destacan tener el control de las evaluaciones en los estudiantes, genera ambientes de aprendizajes amigables, brinda una retroalimentación inmediata comparada con las evaluaciones tradicionales lo que ayuda mejorar el nivel de aprendizaje (Alruwais et al., 2018, Ridgway et al., 2004, Osuji, 2012), es fácil de utilizar, mejora el desempeño académico, incrementa la motivación del estudiante (Eljinini M. y Alsamarai S., 2012, Gilbert et al., 2011, Marriott, 2009).

Adicionalmente, ayuda a los estudiantes que se encuentran en áreas remotas a aprender y evaluar en sus distintas ubicaciones y hacerlo en cualquier momento, lo que brinda flexibilidad a los estudiantes al realizar sus evaluaciones (Williams y Wong, 2009, Kikelomo, 2010). Fomenta el desarrollo de las competencias profesionales en los estudiantes y promueve la retroalimentación prospectiva (Ion et al., 2013).E con respecto a los docentes, el uso de e-evaluaciones ahorrará tiempo al profesor, la evaluación electrónica ayuda al docente a mejorar la calidad de la retroalimentación para los estudiantes (Sorensen, 2013 y Osuji, 2012), permite realizar un seguimiento del rendimiento de los estudiantes, reducir la carga para evaluar a 
Espino Román, P., Olaguez Torres, E., López Hernández, R. A. y Vásquez Martínez C. R. Entorno virtual e-evaluaciones como herramienta de gestión en grupos numerosos

un gran número de estudiantes, mismo que se busca atacar y contribuir con este estudio (Ellaway y Masters, 2008 y Nicol, 2007).

\section{OBJETIVOS}

Así el proyecto consistió en el diseño e implementación de un entorno virtual de aprendizaje mediante el uso de e-evaluaciones basado en herramientas web, que contribuya en la solución al problema de la evaluación y seguimiento del rendimiento del estudiante en los grupos numerosos. Que tendrá como función principal apoyar en el proceso de aprendizaje promoviendo que las evaluaciones y el seguimiento académico de estudiantes se puedan monitorear continuamente durante el desarrollo de su formación académica contribuyendo a la administración de las evaluaciones aplicadas a los estudiantes. Para evaluar el uso del entorno virtual de aprendizaje basado en e-evaluaciones como una alternativa tecnológica, sea limitado el estudio a la unidad académica de ingeniería mecatrónica de la Universidad Politécnica de Sinaloa, México, particularmente en la asignatura de mantenimiento y seguridad industrial, actualmente esta asignatura está conformada por cinco grupos con al menos 40 estudiantes cada uno. Esta experiencia se realizó en el ciclo escolar 2018-2019. Para determinar el impacto que se obtuvo del diseño de entorno virtual se realizó un cuestionario que mide el grado de satisfacción tanto de los estudiantes y docentes considerando la usabilidad, accesibilidad y funcionamiento

\section{METODOLOGÍA}

Existe en el mercado actual un elevado número de plataformas tecnológicas destinadas para llevar a cabo un entorno virtual de enseñanza-aprendizaje a distancia, como por ejemplo: Blackboard, Convene, Embanet, eCollege.com, IntraLearn, Symposium, TopClass, WebCT, TheLearning Manager, WebMentor, Lotus LearningSpace, o LUVIT, (Llorente, 2006). Por tal motivo el propósito de este artículo es desarrollar e implementar una plataforma de entorno virtual de evaluación propia dentro de la misma institución educativa denominado Entorno Virtual e-evaluaciones (EVEE), desarrollado por un equipo multidisciplinario de la Universidad Politécnica de Sinaloa, México.

El diseño del entorno EVEE está basado en el uso de herramientas web, debido a que tienen diversas ventajas entre las que se encuentran: Facilitar el trabajo colaborativo y a distancia puede ser usadas por varios usuarios al mismo tiempo además puede ser alojada en un servidor; Las aplicaciones desarrolladas son dinámicas, atractivas e interactivas. EVEE ayudar tanto al docente como estudiante en la elaboración y respuesta de evaluaciones electrónicas de una manera fácil y sencilla. Por lo que deberá tomar en cuenta las siguientes especificaciones de diseño tales como: Gestión de los usuarios (en sus diferentes roles de administrador, docente, estudiantes); Recursos educacionales requeridos (presentaciones, bibliografías, recursos de información); Actividades de formación, matrícula, control y seguimiento del proceso de evaluaciones, generación de reportes de resultados. 
Espino Román, P., Olaguez Torres, E., López Hernández, R. A. y Vásquez Martínez C. R. Entorno virtual e-evaluaciones como herramienta de gestión en grupos numerosos

Para ello se utilizó Bootstrap un framework desarrollado dentro de la compañía Twitter con el objetivo de estandarizar las herramientas que se utilizan en el desarrollo del front-end. Bootstrap consiste en un conjunto de librerías de CSS encargadas de proporcionar un estilo a las interfaces de las aplicaciones web, así como diversos componentes de JavaScript que se basan en la librería jQuery de JavaScript, los cuales permiten que el diseño de la interfaz sea rápido, fácil y dinámico, ofreciendo diversas ventajas como lo es el responsive web design. El entorno virtual consiste en el uso de CodeIgniter desarrollado por EllisLab, es un framework de desarrollo rápido de software de código abierto utilizado en aplicaciones web dinámico con PHP. CodeIgniter contiene diversas librerías que incentivan al desarrollo de las aplicaciones web de una manera específica permitiendo clasificar los diferentes scripts, así como tener un código limpio y organizado, facilitando la creación y mantenimiento del código. Implementa el proceso de desarrollo conocido como Model View Controller (MVC), que es un estándar de programación de aplicaciones.

En cuanto a la composición de la plataforma se cuenta con 5 módulos principales, que se muestran en la figura 1, los cuales son: cursos, configuración, grupos, usuarios y evaluaciones, el acceso de cada uno de ellos tiene la limitante que ciertos módulos no se encuentran disponibles para todos los niveles de acceso (administrador, docente y estudiante).

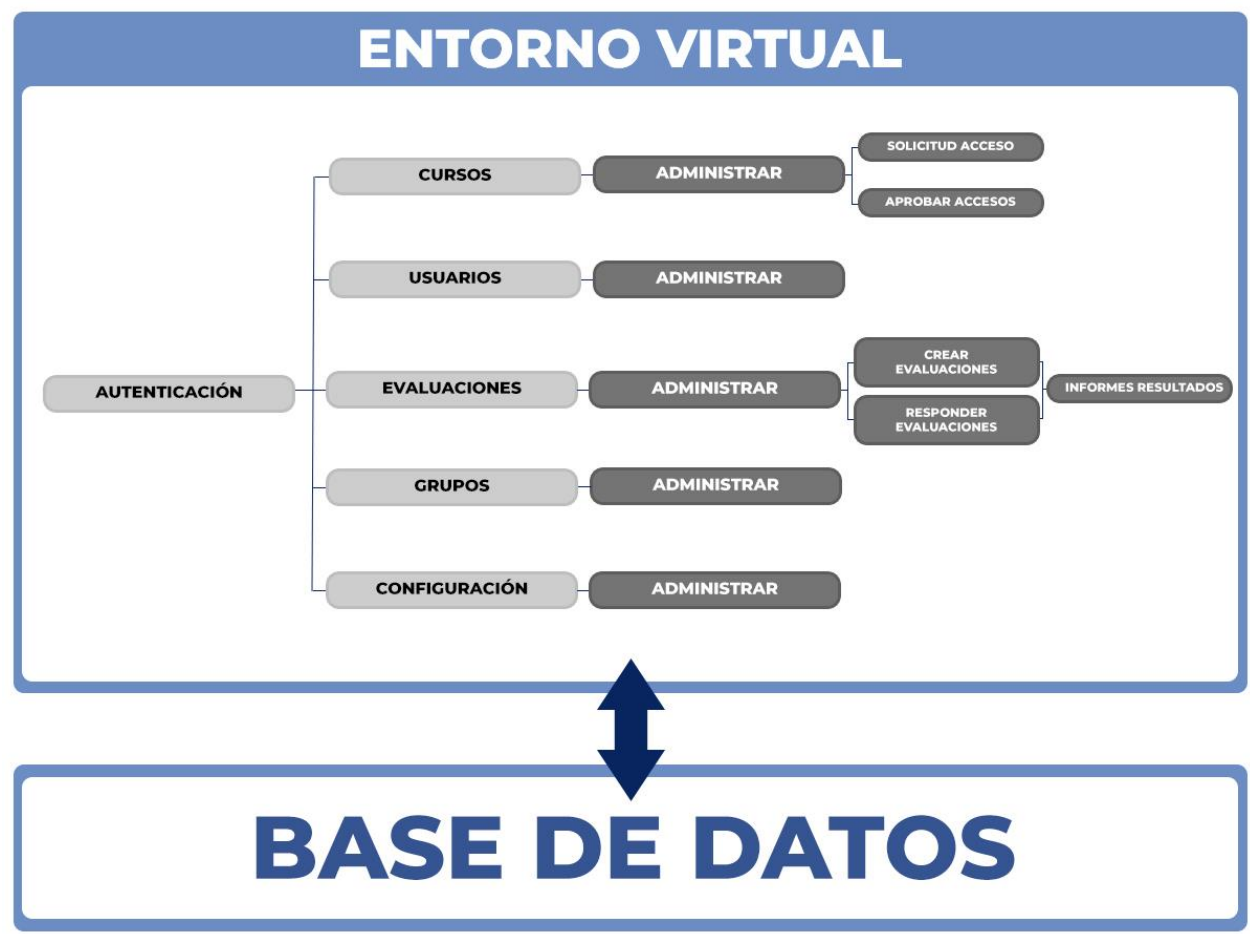

Figura 1: diagrama de componentes de la plataforma.

Fuente: elaboración propia. 
El entorno virtual e-evaluaciones (EVEE), favorece la evaluación continua de los estudiantes en grupos numeroso. El EVEE, permite seleccionar el instrumento de evaluación entre los que se encuentran los siguientes: opción múltiple; preguntas de desarrollo (abiertas); falso y verdadero; resolver problemas con procedimiento y resultado, semejante a una pregunta de desarrollo, solo que la respuesta es numérica y se permite un margen de error. Además, se puede permitir añadir imágenes, recomendar lecturas, recursos de consulta, material de apoyo y con capacidad para recibir archivos en formato PDF, Word, Excel, PowerPoint, por parte del estudiante. Se puede seleccionar que las preguntas sean aleatorias o fijas, así como también que las opciones sean aleatorias. El EVEE permite mostrar los resultados de los estudiantes de forma automática en el entorno virtual y/o descargar un reporte en formato PDF de cada estudiante, posibilitando la evaluación continua. En forma general, el entorno virtual presenta un panel frontal con una interfaz sencilla y fácil de utilizar, debido a que el objetivo es brindar al estudiante y docente un diseño amigable e intuitivo. El primer paso para ingresar al entorno virtual EVEE, es acceder a la página electrónica www.seyferteq.com/evee tanto docentes y estudiantes podrán generar los accesos a la plataforma completando un formulario para matricularse, el sistema le genera un usuario y contraseña, el cual le permite acceder al panel de administración. En la figura 2, se muestra el módulo principal para el proceso de evaluación de los estudiantes en esta sección se realiza el seguimiento de los estudiantes durante el curso en cada una de las asignaturas.

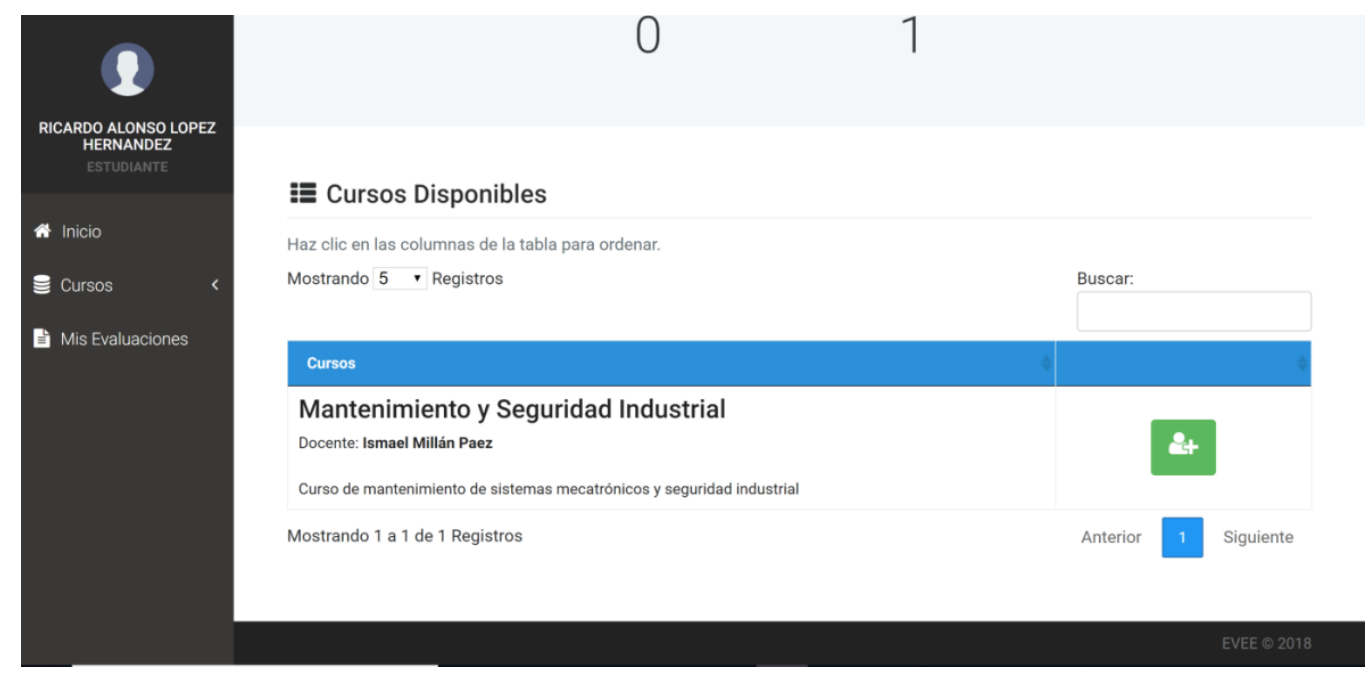

Figura 2: sección de administración de los cursos de los estudiantes. Fuente: elaboración propia.

Una vez registrado el curso, unidades y grupos, la plataforma EVEE, permite al docente crear una evaluación para sus estudiantes dependiendo de la unidad de aprendizaje a evaluar. Para el registro de evaluación se ingresa "Título" el cual consiste en asignarle un nombre a la evaluación, se selecciona el "Curso" que se va a evaluar, así como también la "unidad de aprendizaje" y a su vez seleccionar el grupo de estudiantes que estará disponible la evaluación, se tiene la opción de seleccionar la hora de inicio y fin de la evaluación, así como el rango de fechas en la que la 
Espino Román, P., Olaguez Torres, E., López Hernández, R. A. y Vásquez Martínez C. R. Entorno virtual e-evaluaciones como herramienta de gestión en grupos numerosos

evaluación estará disponible. El docente crea el procedimiento de evaluación de sus estudiantes seleccionado el instrumento de evaluación entre preguntas aleatorias o fijas, valora cuantitativa y cualitativamente a los estudiantes con calificaciones y comentarios de las actividades realizadas, los resultados son mostrados dentro del mismo entorno virtual y/o importa las notas de los cuestionarios/evaluaciones realizadas para su descarga de un reporte en formato PDF, ver figura 3.

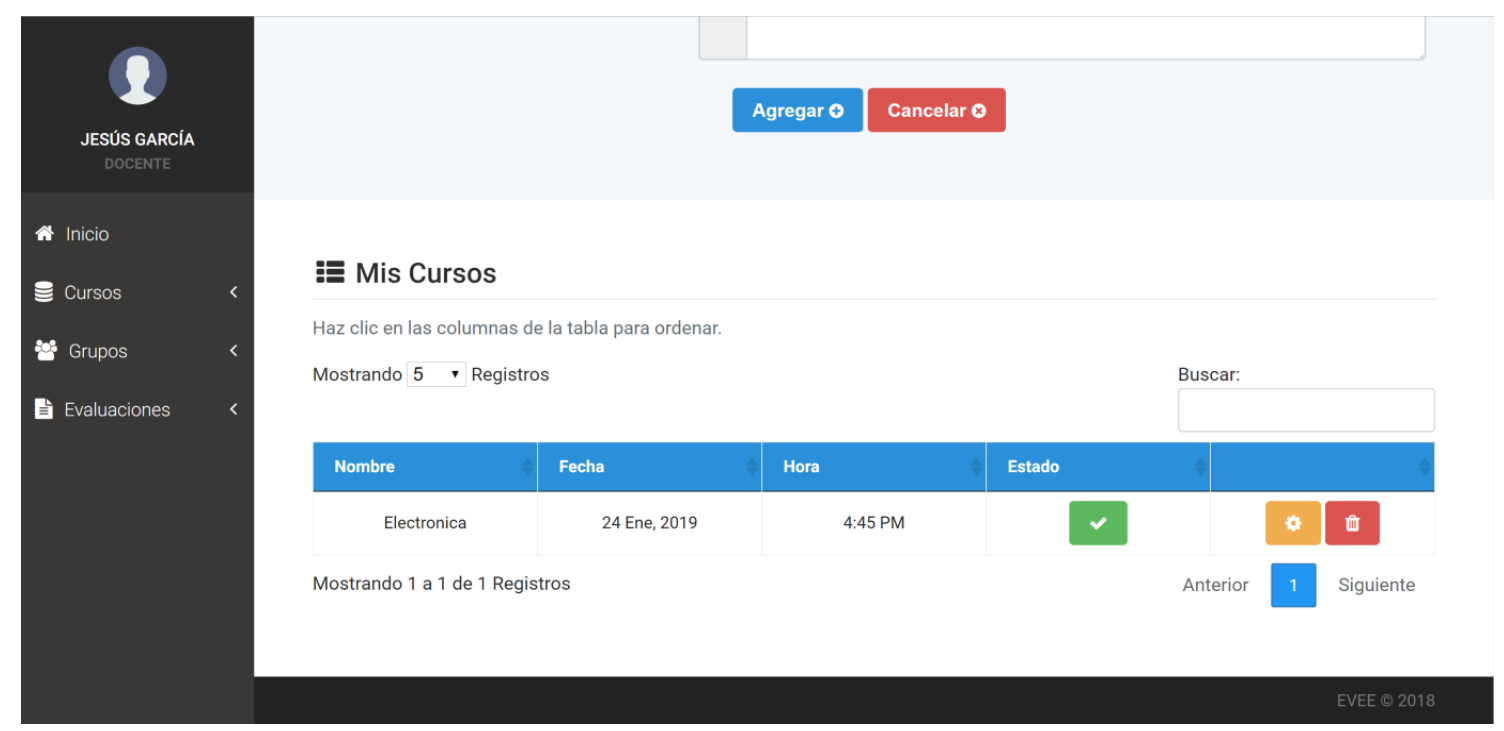

Figura 3: sección de administración de los cursos del docente.

Fuente: elaboración propia.

En la sección de "Mis Evaluaciones", el estudiante puede consultar las evaluaciones que se encuentran disponibles para contestar, o para consultar los resultados de las evaluaciones que ya completó. Por cada evaluación disponible, se presentan dos opciones, una es acceder a la evaluación y la segunda opción es visualizar los resultados una vez que la evaluación ya fue completada, ver figura 4, en la figura 5, se muestran los resultados obtenidos por el estudiante y la retroalimentación de las respuestas no aceptadas. 
Espino Román, P., Olaguez Torres, E., López Hernández, R. A. y Vásquez Martínez C. R. Entorno virtual e-evaluaciones como herramienta de gestión en grupos numerosos

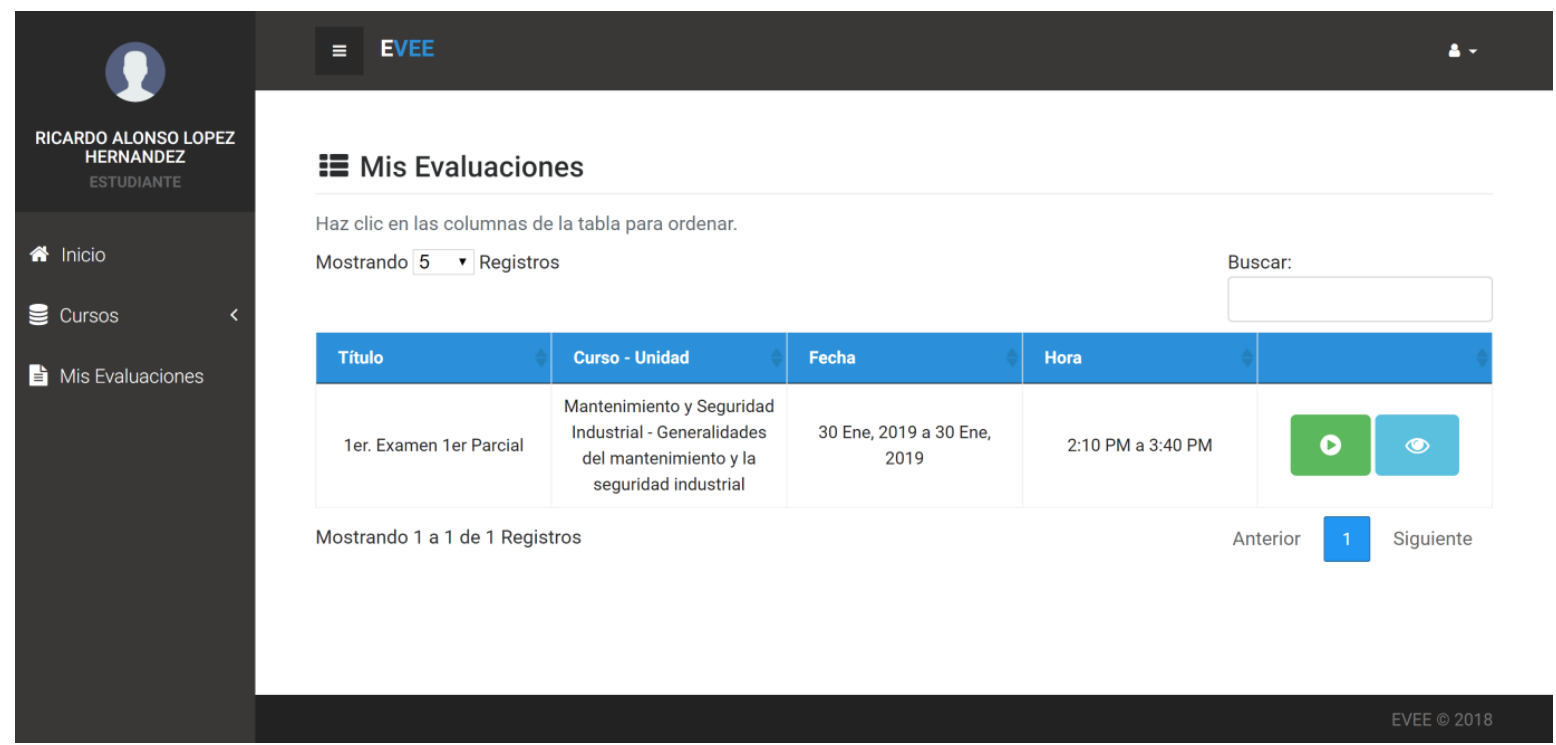

Figura 4: sección de administración de los cursos del docente.

Fuente: elaboración propia.

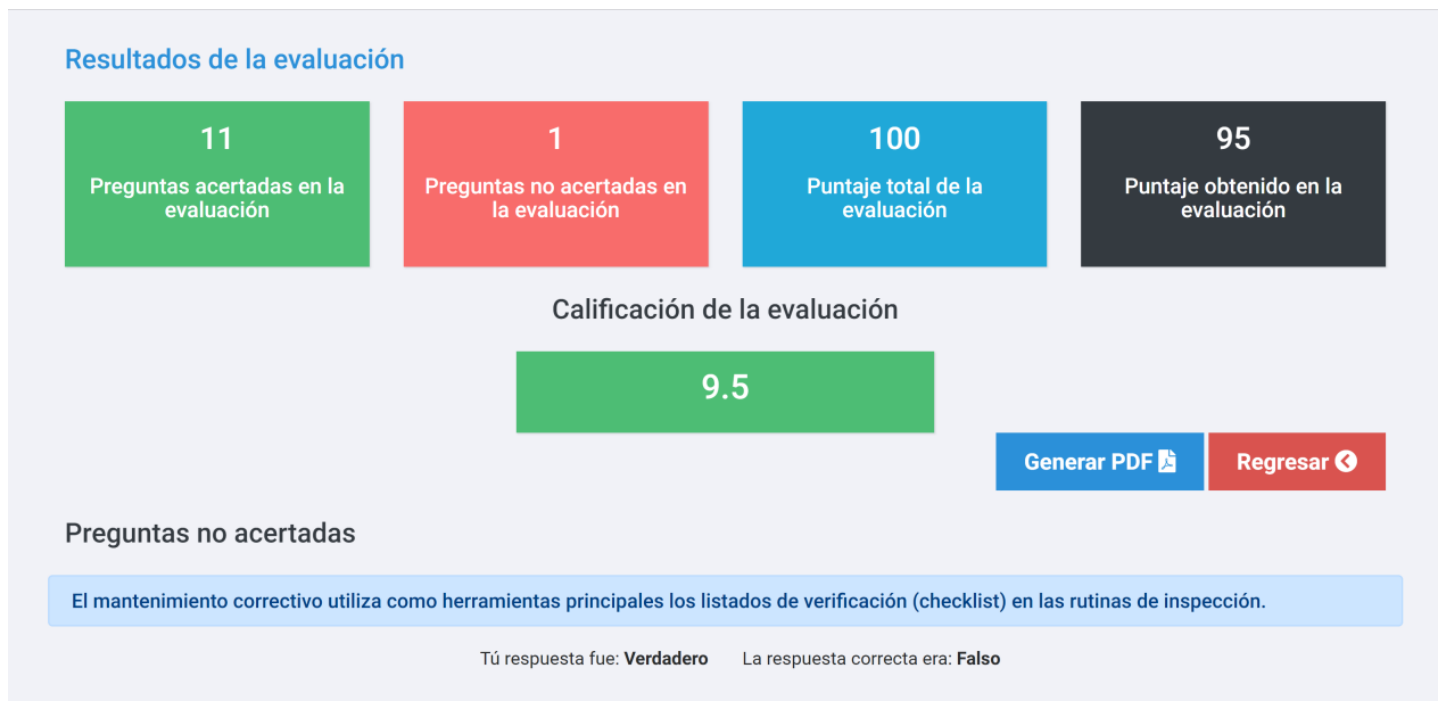

Figura 5: sección los resultados de la evaluación presentada por los estudiantes. Fuente: elaboración propia.

Una vez que los estudiantes concluyen y dan por terminada la evaluación, los docentes pueden obtener un reporte general de la evaluación, en la figura 6, se muestran los datos generales de la evaluación, el número de estudiantes que contestaron la evaluación, número de estudiantes que aprobaron la actividad, número de estudiantes que no aprobaron, así como el promedio general del grupo en la evaluación, la plataforma muestra los resultados dentro de la misma aplicación además de permitir importar los resultados de la evaluación en archivo PDF. 
Espino Román, P., Olaguez Torres, E., López Hernández, R. A. y Vásquez Martínez C. R. Entorno virtual e-evaluaciones como herramienta de gestión en grupos numerosos

Resultados de la evaluación:

1er. Examen 1er Parcial

Mantenimiento y Seguridad Industrial | Generalidades del mantenimiento y la seguridad industrial | mecatrónica 2-5

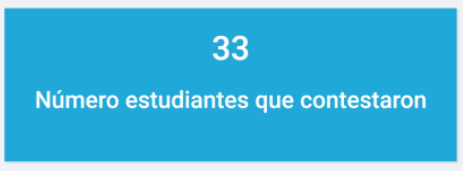

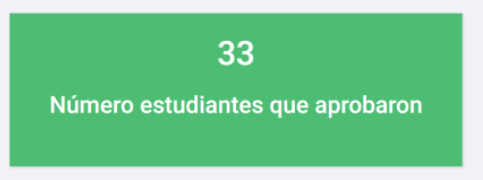

Promedio General

\section{4}

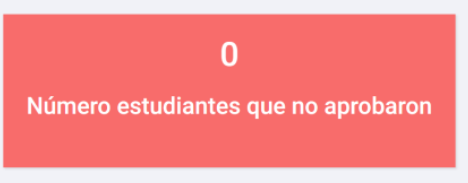

Generar PDF 2

\section{Regresar $\odot$}

Figura 6: pantalla de los resultados de una evaluación presentada a los docentes.

Fuente: elaboración propia.

\section{RESULTADOS}

Con el propósito de evaluar el uso de la plataforma virtual EVEE, fue necesario realizar un cuestionario como instrumento de recopilación que fue aplicada para los docentes y estudiantes para determinar: usabilidad, accesibilidad y funcionamiento. En este estudio se describen los datos obtenidos en el análisis descriptivo consistente en el empleo de medidas de tendencia central y de frecuencias para clasificar los datos acerca del tema de trabajo y determinar el alcance de los objetivos planteados al inicio de este proceso. El cuestionario fue enviado a los docentes y estudiantes a través del uso de la herramienta de Google "Formularios de Google", el cual permite enviar la encuesta y recopilar información de forma fácil y eficiente.

a)-Evaluación por parte de los docentes a la plataforma virtual EVEE:

Se seleccionó a un grupo de 31 docentes quienes representan el 100\% de docentes que imparten 15 o más horas a la semana en la unidad académica de ingeniería mecatrónica, de los cuales el $60 \%$ es masculino y un $40 \%$ femenino. El rango de edad fue de entre 23 a 55 años. En tipo de nombramiento contractual de los docentes, corresponde al 77\% Profesor Tiempo de Completo y 23\% a docentes de Asignatura. De acuerdo con los resultados de la encuesta aplicada a los docente, el uso de la plataforma resulta una experiencia novedosa e innovadora para los docentes; se les cuestionó ¿El diseño de la interfaz de usuario permite flexibilidad de acomodo del acceso y navegación? El 100 \% de los docentes consideraron "Excelente" el diseño de la interfaz de usuario, es decir el acceso y registro a la plataforma virtual no presentó problemas para ello.

En cuanto a la estructura de la interfaz, ¿Se encuentran organizados de una forma consistente, por ejemplo, cursos, evaluaciones, seguimiento de estudiantes, unidades de aprendizaje con las siguientes secciones: introducción, contenidos, recursos, tareas y evaluación?, el 100\% de los docentes lo evaluaron "Excelente". ¿Los contenidos tienen una estructura bien definida lineal y jerárquica? el $75 \%$ de los docentes 
Espino Román, P., Olaguez Torres, E., López Hernández, R. A. y Vásquez Martínez C. R. Entorno virtual e-evaluaciones como herramienta de gestión en grupos numerosos

consideró "Excelente". Se les cuestionó ¿El diseño de los contenidos permite la interactividad a través de una navegación autónoma?, el 75\% lo evaluó como "Excelente", mientras que el 25\% lo considero "Bueno".

¿El lenguaje utilizado para el desarrollo de los contenidos es adecuado?, "Excelente" con el 50\% y "Bueno" con el 50\%. El 100\% de los docentes manifestaron como "Excelente" que la plataforma virtual permite el acceso desde cualquier navegador: internet Explorer, Mozilla Firefox, Google Chrome. El 50\% indicó como "Excelente" la información que se proporciona sobre el perfil del docente, seguido por el 25\% lo consideró "Bueno" y "Suficiente". Ante la posibilidad de que la plataforma permite establecer fechas límites de actividades para desarrollar por parte de los estudiantes, el 25\% selecciono "Excelente" y otro 75\% indico como "Bueno". La plataforma virtual EVEE proporciona al docente una gran variedad de opciones de seleccionar un instrumento de evaluación para sus estudiantes por lo que se les cuestionó ¿Los instrumentos de evaluación disponibles en la plataforma virtual favorecen la evaluación continua en grupos numerosos?, 75\% lo consideró "Excelente" seguido de "Bueno" con $25 \%$. En la figura 7 se muestra el concentrado de respuesta de la pregunta 1 a la 9.

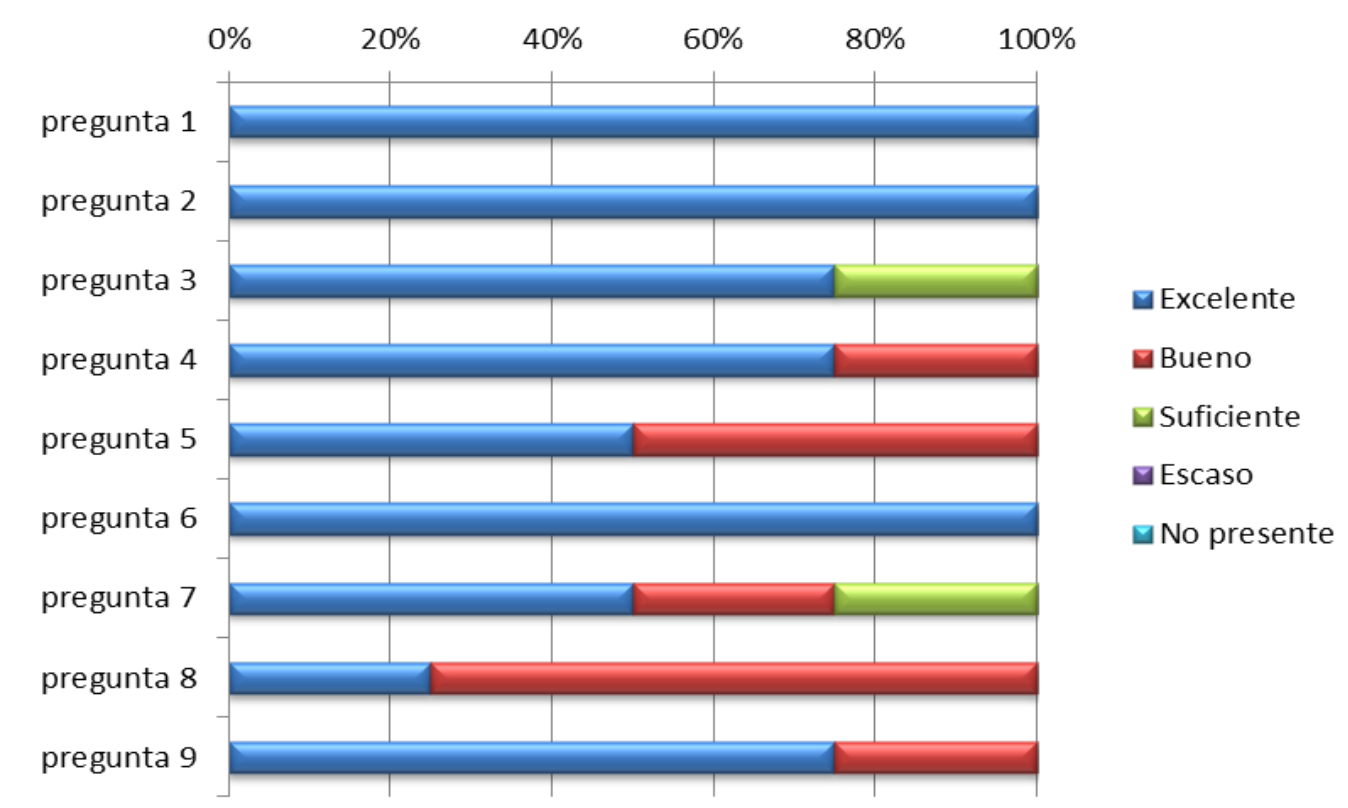

Figura 7: respuestas proporcionadas por los docentes de la pregunta 1 a la 9. Fuente: elaboración propia.

Además se le cuestionó a los docentes ¿Cuánto es el tiempo que destinan para evaluar de forma tradicional un examen en un grupo de 40 estudiantes o más? Siendo la respuesta de "3 o más horas" con mayor frecuencia entre los docentes con el $50 \%$, seguida con el $30 \%$ de " 1 a 2 horas", y con menor frecuencia de " 2 a 3 horas" con el $20 \%$, ver figura 8 . 
Espino Román, P., Olaguez Torres, E., López Hernández, R. A. y Vásquez Martínez C. R. Entorno virtual e-evaluaciones como herramienta de gestión en grupos numerosos

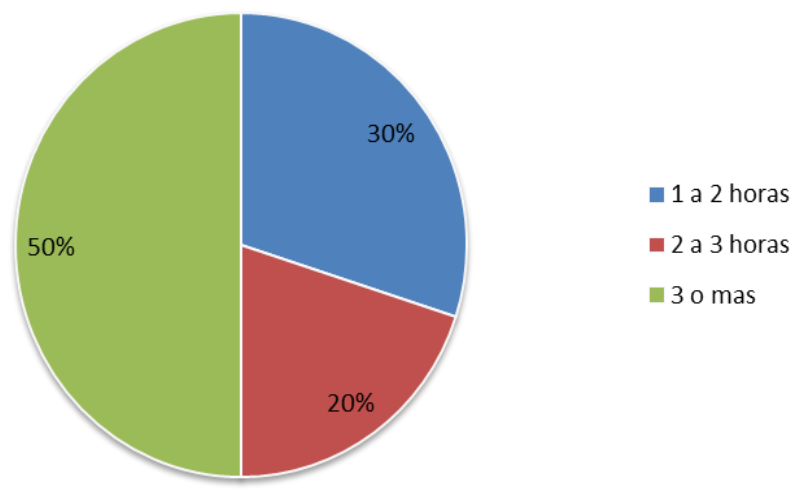

Figura 8: respuestas a la pregunta ¿Cuánto es el tiempo que destinan para evaluar de forma tradicional un examen en un grupo de 40 estudiantes o más?

Fuente: elaboración propia.

Por último se les cuestionó a los docentes de que con base a su experiencia ¿Cuál es el tiempo promedio que le lleva a un estudiante realizar un examen de forma tradicional?, la respuesta con mayor frecuencia fue "1 hora o más" con el 65\%, mientras que "30 minutos a 1 hora" obtuvo el $35 \%$ y la respuesta " 0 a 30 minutos" no tuvo ninguna mención, ver figura 9.

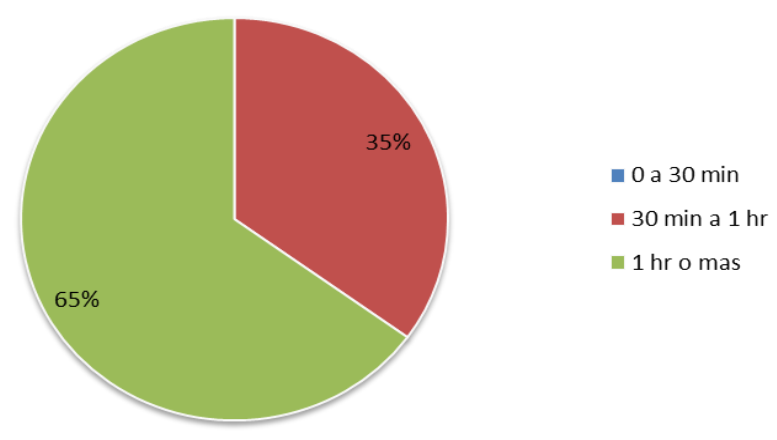

Figura 9: respuestas ¿Cuál es el tiempo promedio que le lleva a un estudiante realizar un examen de forma tradicional?

Fuente: elaboración propia.

b)- Evaluación del uso dela plataforma virtual EVEE por parte de los estudiantes:

El instrumento para evaluar el uso de la plataforma virtual EVEE aplicado a los estudiantes se utilizó el presentado por Nass et al. (2017) en su trabajo de investigación. Se seleccionó un grupo de 40 estudiantes de la asignatura de mantenimiento y seguridad industrial de segundo cuatrimestre de la unidad académica de ingeniería mecatrónica, representado por $70 \%$ masculinos y el 30\% femeninos, con un rango de edad de 18 a 23 años. La escala adoptada para la evaluación es la siguiente: muy en desacuerdo, en desacuerdo, ni de acuerdo ni en 
Espino Román, P., Olaguez Torres, E., López Hernández, R. A. y Vásquez Martínez C. R. Entorno virtual e-evaluaciones como herramienta de gestión en grupos numerosos

desacuerdo, de acuerdo, muy de acuerdo. De acuerdo con los resultados del cuestionario, la plataforma virtual EVEE resulta una gran experiencia y de gran ayuda para los estudiantes. El 75\% de los estudiantes consideraron estar "Muy de acuerdo" en la presentación de la plataforma mientras que el 25\% consideró estar "De acuerdo" con ello.

En cuanto a la interactividad y navegación en el prototipo hacen más fácil y entretenidas las evaluaciones el 50\% de los estudiantes estuvieron "Muy de acuerdo" mientras que el $45.8 \%$ estuvo "De acuerdo" y solo el $4.2 \%$ mencionó estar "Ni de acuerdo ni en desacuerdo". El menú principal y los enlaces son orientadores en la navegación dentro del prototipo un 50\% mencionó estar "Muy de acuerdo" mientras que el 50\% restante estuvo "De acuerdo". En cuanto a que si les gustaría que la plataforma incluyera mayor cantidad de contenidos el 37.5\% estuvo "Muy de acuerdo", el 33.3\% se decantó por mencionar que estaba "De acuerdo" mientras que el 29.2\% mencionó estar "Ni de acuerdo ni en desacuerdo". Acerca sobre si esta plataforma virtual hace que los contenidos adquieran un excelente grado de claridad el $45.8 \%$ de los estudiantes estuvieron "De acuerdo", el 37.5\% señalo que "Muy de acuerdo", el 12.5\% en "Ni de acuerdo ni en desacuerdo" y por último, un $4.2 \%$ "En desacuerdo". Al cuestionarles si el material propicia la participación de los estudiantes el 58.3\% estuvo "De acuerdo", el 37.5\% "Muy de acuerdo" y solo un 4.2\% "En desacuerdo". Y, por último, se les cuestionó acerca del agrado de la forma como esta plataforma me impulsa a seguir en mi proceso de aprendizaje el $66.7 \%$ estuvo "Muy de acuerdo" mientras que el 33.3\% mencionó estar "De acuerdo", en la figura 10 , se muestra la respuesta de los estudiantes.

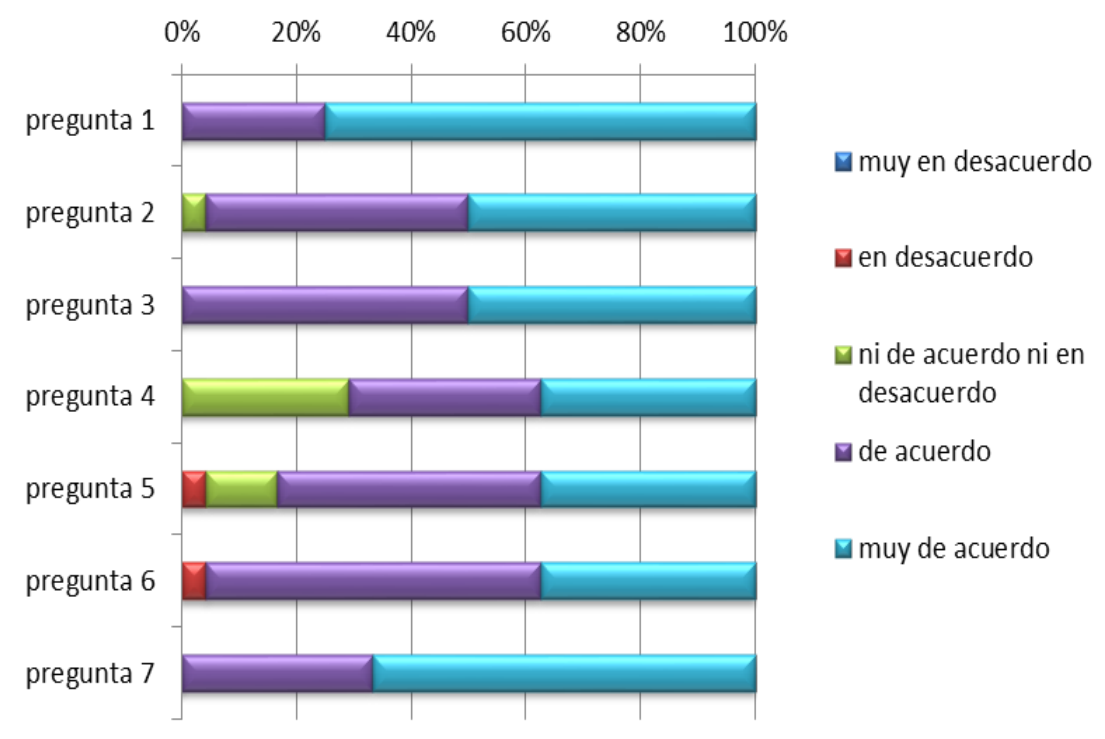

Figura 10: respuestas proporcionadas por los estudiantes.

Fuente: elaboración propia.

De los resultados obtenidos en esta investigación, se puede deducir que los docentes se manifestaron que el entorno virtual de e-evaluaciones presenta una 
Espino Román, P., Olaguez Torres, E., López Hernández, R. A. y Vásquez Martínez C. R. Entorno virtual e-evaluaciones como herramienta de gestión en grupos numerosos

buena estructura para poder crear contenido, el diseño de la interfaz del entorno es intuitivo lo que la hace sencilla y fácil de operar, por lo tanto, facilita la navegación dentro de ella. También destacan la portabilidad de EVEE, debido a su diseño y desarrollo, se puede utilizar en dispositivos móviles sin dificultad alguna y sin ninguno tipo de descarga alterna, los instrumentos de evaluación con los que cuenta el entorno virtual son adecuados para crear y aplicar evaluaciones. En cuanto a los estudiantes, se puede inferir que el uso de EVEE fue una buena experiencia debido a que se una alternativa diferente para poder contestar evaluaciones y obtener una retroalimentación en el instante, a su vez, la interactividad y navegación en la plataforma hacen más fácil y entretenidas las evaluaciones. Se observó que con el uso del entorno virtual EVEE, se mejoró el tiempo destinado a realizar la evaluación de los docentes ya que en un grupo de 40 estudiantes el tiempo promedio para obtener la evaluación de todo el grupo fue de 21 minutos, bastante significativo si se compara con "3 o más horas" que los docentes señalaron anteriormente.

\section{DISCUSIÓN}

Se llegó a las siguientes conclusiones, considerando que la plataforma está en proceso y constante desarrollo, hasta el momento se puede apreciar que: 1 . El factor tiempo en la aplicación, desarrollo, evaluación y retroalimentación del proceso de evaluación resultó para los docentes prioritario haciendo uso eficiente y estratégico con un mayor margen para la evaluación masiva; 2 . Al referir una optimización en el tiempo de evaluación, el docente dispone de información oportuna para la procedente interpretación y análisis del proceso de aprendizaje basándose en las respuestas e inferencias de sus estudiantes, proporcionando herramientas para promover la realimentación y corregir errores que propician el lento o bajo entendimiento de cierta competencia o disciplina según sea la materia en curso; 3 . El desarrollo de la plataforma con la característica de la e-evaluaciones aporta elementos de apoyo didáctico al estudiante, promoviendo el aprendizaje autodidacta y promoviendo la realimentación por parte del docente que determina la evaluación; 4. La plataforma se desarrolla y presenta una interfaz amigable esta característica le atribuye cierta confiabilidad a los usuarios docente y discente (estudiante), aportando un área de oportunidad para el desarrollo en formato aplicación y que cumpla el mismo propósito como herramienta de apoyo para el fortalecimiento de las competencias docentes y el proceso de aprendizaje en el estudiante.

\section{REFERENCIAS}

Aguerrondo, I. (2008). La influencia del contexto en la efectividad de la escuela: Consideraciones para el desarrollo profesional docente. R. Blanco (Eds.), Eficacia escolar y factores asociados en América Latina y el Caribe (pp. 61). Chile: Salesianos Impresores S.A.

Alruwais, N.; Wills, G. \& Wald, M. (2018). Advantages and Challenges of Using eAssessment. International Journal of Information and Education Technology, 8(1), 3437. doi: https:// doi.org/10.18178/ijiet.2018.8.1.1008 
Espino Román, P., Olaguez Torres, E., López Hernández, R. A. y Vásquez Martínez C. R. Entorno virtual e-evaluaciones como herramienta de gestión en grupos numerosos

Arias, L.; Arias, E. \& Arias, J. (2013). La educación superior en México: financiamiento de instituciones y estudiantes. Tecsistecatl, 4(13), 1-16.

Bretones, A. (2008). Participación del alumnado de Educación Superior en su evaluación. Revista de Educación, (347), 181-202.

Cano, C. y Hernández, S. (2009). La evaluación del aprendizaje en ambientes virtuales. X congreso nacional de investigación educativa, área 7: entornos virtuales de aprendizaje. Recuperado de https://bit.ly/2E9I2IE

Chiva. I.; Ramos, G.; Gómez, M. B. y Alonso, A. (2013). La e-Evaluación del aprendizaje a través de la plataforma Aula Virtual de la Universitat de València. @tic. Revista d'innovació educativa, (11), 60-68.

doi: https:// doi.org/10.7203/attic.11.3051

Cuesta, I. y Alegre, J. (2011). Uso de la plataforma Moodle como herramienta para la evaluación continua de estudiantes en el espacio europeo de educación superior. Vivat Academia Revista de Comunicación, 417-428.

doi: https:// doi.org/10.15178/va.2011.117E.417-428

Eljinini, M.; Alsamarai, S.; Hammed, S. \& Amawi, A. (2012). The impact of eassessments system on the success of the implementation process, International Journal of Modern Education and Computer Science, 4(11), 76-84.

doi: https://doi.org/10.5815/ijmecs.2012.11.08

Ellaway, R. \& Masters, K. E. N. (2008). AMEE guide AMEE guide 32: E-learning in medical education part 1: Learning, teaching and assessment. Medical Teacher, 30(5), 455-73. doi: https://doi.org/10.1080/01421590802108331

Espino, P.; Olaguez, E. \& Hernández, R. (2019). Virtual Learning Environments as a Continuous Assessment Tool in University Students. In L. Bailey (Ed.), Educational Technology and the New World of Persistent Learning (pp. 229-252). Hershey, PA: IGI Global. doi: https:// doi.org/10.4018/978-1-5225-6361-7.ch012

Fondón, I.; Madero, M. J. y Sarmiento, A. (2010). Principales Problemas de los Profesores Principiantes en la Enseñanza Universitaria. Formación Universitaria, 3(2), 21-28. doi: https://doi.org/10.4067/S0718-50062010000200004

Gilbert, L.; Whitelock, D. \& Gale, V. (2011). Synthesis report on assessment and feedback with technology enhancement. Recuperado de https:// bit.ly/2AjbF81

González, H. (2015). Cobertura en educación superior: de los compromisos a la realidad. Recuperado de https://bit.ly/2RXTHkw

Gutiérrez, N.; Castro, A. y Segure, T. (2000). El número de alumnos por curso y su relación con la calidad de los procesos pedagógicos. Docencia, (11), 37-44. 
Espino Román, P., Olaguez Torres, E., López Hernández, R. A. y Vásquez Martínez C. R. Entorno virtual e-evaluaciones como herramienta de gestión en grupos numerosos

Hernández, N.; Muñoz, P. C. y González, M. (2018). La E-Evaluación en el Trabajo Colaborativo en Entornos Virtuales: Análisis de la Percepción de los Estudiantes. Edutec. Revista Electrónica de Tecnología Educativa, (65), 16-28. doi: https:// doi.org/10.21556/edutec.2018.65.997

Ion, G.; Silva, P. y García, E. (2013). El feedback y el feedforward en la evaluación de las competencias de estudiantes universitarios. Revista Profesorado, 17(2), 287-301.

Kikelomo, M. A.; Wills, G. \& Argles, D. (2010). User Security Issues in Summative EAssessment Security. International Journal of Digital, 1(2), 135-147.

Lara, L. R. (2001). El dilema de las teorías de enseñanza-aprendizaje en el entorno virtual. Comunicar, (17), 133-136.

Lorente, M. C. (2007). Moodle como entorno virtual de formación al alcance de todos. Comunicar, (28), 197-202.

Martínez, N. L.; Galindo, R. M. y Galindo, L. (2013). Entornos virtuales de aprendizaje abiertos; y sus aportes a la educación. Recuperado de https://bit.ly/2J59ZyZ

Martínez, F. (2013). Dificultades para implementar la evaluación formativa. Perfiles Educativos, 15(139), 128-150.

Marriott, P. (2009). Students' evaluation of the use of online summative assessment on an undergraduate financial accounting module. British Journal of Educational Technology, 40(2), 237-254. doi: https://doi.org/10.1111/j.1467-8535.2008.00924.x

Mendoza, L. V. (2013). Sobrepoblación Estudiantil y Desempeño Docente En El Aula. Quetzaltenango: Guatemala. Recuperado de https://bit.ly/2RjkSRy

Murrieta, R. (2016). Entornos virtuales de aprendizaje en educación superior: una experiencia con el uso del Moodle. Investigación Cualitativa en Educación, (1), 12621665.

Nass, L. S.; Mendoza, M. A., Millanao, L. E. \& Ortega, R. M. (2017). Assessment of an educational platform at the University of Concepción. Revista Cubana de Educación Medica Superior, 31(1), 99-113.

Nicol, D. (2007). E-assessment by design: Using multiple-choice tests to good effect. Journal of Further and Higher Education, 31(1), 53-64. doi: https:/ / doi.org/10.1080/03098770601167922

Osuji, U. (2012). The use of e-assessments in the Nigerian higher education system.The Turkish Online Journal of Distance Education, 13(1), 140-152. 
Espino Román, P., Olaguez Torres, E., López Hernández, R. A. y Vásquez Martínez C. R. Entorno virtual e-evaluaciones como herramienta de gestión en grupos numerosos

Ridgway, J., McCusker, S. \& Pead, D. (2004). Literature review of e-assessment. Futurelab. Recuperado de https:// bit.ly/2MHmQKO

Rocha, A. M. (2017). Estrategias pedagógicas para aulas numerosas un reto docente. Revista Electrónica TicALS, 1(3), 99-120.

Rodríguez, A. y Díaz, I. (2015). Estrategias y técnicas docentes para aplicar en clases magistrales y trabajo en equipo con grupos grandes de alumnos universitarios. Ikastorratza, e-Revista de didáctica, (14).

Rodríguez, G.; Ibarra, M. S. y Gómez, M. A. (2011). E-Autoevaluación en la Universidad: un reto para profesores y estudiantes. Revista de Educación, (356), 401430. doi: https:/ / doi.org/10-4438/1988-592X-RE-2010-356-045

Salinas, M. I. (2011). Entornos virtuales de aprendizaje en la escuela: tipos, modelo didáctico y rol de docente. Recuperado de https:// bit.ly/2PtBfOV

Santibáñez, J. (2010). Aula virtual y presencial en aprendizaje de comunicación audiovisual y educación. Comunicar, 35(18), 183-191.

doi: https://doi.org/10.3916/C35-2010-03-12

Secretaría de Educación Pública (2016). Estadística del sistema educativo Sinaloa ciclo escolar 2015-2016. Recuperado de https://bit.ly/2GIWbMD

Secretaría de Educación Pública (2017). Comunicado 249. México logra progresos notables en educación preescolar, técnica y superior: OCDE. Recuperado de https://bit.ly/2RXA07n

Sobrados, M. (2016). El trabajo docente en grupos numerosos. Experiencias en el uso del portafolio. Opción, 32(10), 773-788.

Sorensen, E. (2013). Implementation and student perceptions of e-assessment in a chemical engineering module. European Journal of Engineering Education, 38(2), $172-$ 185. doi: https:// doi.org/10.1080/03043797.2012.760533

Universidad Politécnica de Sinaloa. (2017). Agenda Estadística Universidad Politécnica de Sinaloa Ciclo Escolar 2015-2016. Recuperado de https://bit.ly/2SNunx1

Wambui, J.; Luvanda, A.; Matende, S. \& Kamundi, S. (2014). Impersonation Challenges Associated With E-Assessment of University Students. Journal of Information Engineering and Applications, 4(7), 60-68.

William, J. B. \& Wong, A. (2009). The efficacy of final examinations: A comparative study of closed-book, invigilated exams and open-book, open-web exams.British Journal of Educational Technology, 40(2), 227-236.

doi: https://doi.org/10.1111/j.1467-8535.2008.00929.x 
Espino Román, P., Olaguez Torres, E., López Hernández, R. A. y Vásquez Martínez C. R. Entorno virtual e-evaluaciones como herramienta de gestión en grupos numerosos

\section{AUTORES:}

\section{Piero Espino Román}

Director de la unidad académica de ingeniería mecatrónica de la Universidad Politécnica de Sinaloa (México). Líder del cuerpo académico Diseño y Tecnología Mecatrónica, líneas de investigación; Diseño de Sistemas para Aplicaciones Mecatrónicas y Mecatrónica Educativa.

pespino@upsin.edu.mx

Orcid ID: https:// orcid.org/0000-0002-1235-0134

Scopus ID: 56712818900

Researchgate: https://www.researchgate.net/profile/Piero_Espino

\section{Eugenia Olaguez Torres}

Coordinadora de Posgrado Maestría en Enseñanza de las Ciencias de la Universidad Politécnica de Sinaloa (México). Miembro del Núcleo Académico Básico del programa de Maestría en Enseñanza de las Ciencias. Miembro del Sistema Nacional de Investigadores Nivel C.

jolaguez@upsin.edu.mx

Orcid ID: https:// orcid.org/0000-0002-7524-2675

Scopus ID: 56712852800

Researchgate: https://www.researchgate.net/profile/Eugenia_Olaguez

\section{Ricardo Alonso López Hernández}

Egresado de la maestría en ciencias aplicadas de la Universidad Politécnica de Sinaloa (México). Sus intereses en la investigación son el diseño de plataformas web e ingeniería de software.

2016030975@upsin.edu.mx

Orcid ID: https://orcid.org/0000-0002-2364-6969

\section{Claudio Rafael Vásquez Martínez}

Profesor Investigador Titular C, Máxima Categoría, Campus Puerto Vallarta, Centro Universitario de la Costa, Departamento de Ciencias Exactas, Universidad de Guadalajara (México). Líder del Proyecto Matematización Física de la Pintura. Líneas de investigación: Matematización Física de la Pintura, Rocrematica Educativa, Tecnología y Didáctica Matemática Educativa, Educación Especial en Pintura Educativa.

crvasquezm@gmail.com

Orcid ID: https:// orcid.org/0000-0001-6383-270X 